BULLETIN OF THE

AMERICAN MATHEMATICAL SOCIETY

Volume 80, Number 5, September 1974

\title{
THE ZETA FUNCTION OF A CLASS OF RATIONAL VARIETIES
}

\author{
BY STUART TURNER
}

Communicated by A. Mattuck, January 29, 1974

Let $k$ be a field and $V$ be an algebraic $k$-scheme of dimension $n-1$. $V$ is called a Severi-Brauer $k$-scheme if there exists a separable algebraic extension $L / k$ such that $V \times_{k} L$ and $P_{n-1}(L)=\operatorname{Proj} L\left[X_{1}, \cdots, X_{n}\right]$ are isomorphic as $L$-schemes [7]. $V$ is said to be split by $L / k . V$ is called a trivial Severi-Brauer $k$-scheme if $V$ and $P_{n-1}(k)$ are isomorphic as $k$ schemes.

Let $K / k$ be a finite Galois extension and let $G=\mathrm{Gal}(K / k)$. The isomorphism classes (as $k$-schemes) of Severi-Brauer $k$-schemes of dimension $n-1$ which are split by $K / k$ are in canonical one-one correspondence with the elements of the cohomology set $H^{1}(G, P G L(n, K))$ [7]. The exact sequence $1 \rightarrow K^{*} \rightarrow G L(n, K) \rightarrow P G L(n, K) \rightarrow 1$ induces a map

$$
\Delta: H^{1}(G, P G L(n, K)) \rightarrow H^{2}\left(G, K^{*}\right) .
$$

$\Delta$ is injective and $\operatorname{Im} \Delta$ is described as follows: let $\gamma \in H^{2}\left(G, K^{*}\right)$ and let $D(\gamma)$ denote the central division algebra over $k$ defined by $\gamma$, let $[D(\gamma): k]=d^{2}$, then $\gamma \in \operatorname{Im} \Delta$ if and only if $d \mid n$ [6]. Assume now that $\gamma \in \operatorname{Im} \Delta$ and let $\beta=\Delta^{-1}(\gamma)$, then the Severi-Brauer $k$-scheme $V(\beta)$, defined by $\beta$, is isomorphic as a $k$-scheme to the Grassmann variety of left ideals of rank $n$ in the matrix algebra $A=M_{n / d}(D(\gamma))$. Actually, $A$ does not depend on $K$ but only on $n$ and the image of $\gamma$ in $\operatorname{Br}(k)$, the Brauer group of $k$. It was Chatelet [5] who first defined Severi-Brauer varieties and established their basic properties over fields of characteristic zero. In particular, he determined the function field, $K(V(\beta))$, of $V(\beta)$. Amitsur [3] interpreted Chatelet's work from another point of view, obtained new results, and extended Chatelet's theorems to fields of arbitrary characteristic. Today, his determination of $K(V(\beta))$ is more accessible than Chatelet's. The defining relations for $K(V(\beta))$ are simplest when $A$ is a cyclic algebra [1], [2], i.e. there exists a cyclic splitting field $k^{\prime} / k$ for $A$ with $\left[k^{\prime}: k\right]=n$. In this case, let $z \in k^{\prime}$ such that $k^{\prime}=k(z)$.

AMS (MOS) subject classifications (1970). Primary 14D10, 14G10; Secondary $12 \mathrm{~A} 80$.

Key words and phrases. Zeta function, Severi-Brauer scheme. 
Chatelet and Amitsur proved that there exists a $\theta \in k^{*}(\theta$ is determined up to an element of $\left.N_{k^{\prime} / k}\left(k^{\prime}\right)\right)$ such that $K(V(\beta))$ is $k$-isomorphic to the quotient field of $M=k\left[X_{0}, \cdots, X_{n}\right] / I$, where $I$ denotes the ideal generated by

$$
\prod_{g \in \operatorname{Gal}\left(k^{\prime} / k\right)}\left({ }_{0}+g(z) X_{1}+g\left(z^{2}\right) X_{2}+\cdots+g\left(z^{n-1}\right) X_{n-1}\right)-\theta X_{n}^{n}
$$

In this note we are interested in Severi-Brauer schemes over two types of fields: first, $k$, a locally compact field, distinct from $\boldsymbol{R}$ or $\boldsymbol{C}$, of arbitrary characteristic (a $p$-field in the terminology of [11]), and, secondly, $k$, an algebraic number field. In both cases we state results about "models" for Severi-Brauer schemes defined over the ring of integers of $k$ and the specializations of these models. For $k$, a $p$-field, we give the zeta function for the closed fiber of the models described. For $k$, an algebraic number field, and $n$, an odd prime, we give the global zeta function for the model described and its functional equation. Proofs of these results will appear in a later publication. The larger part of this work was done at The Institute for Advanced Study during 1970-1972 where the author profited from A. Weil's advice and encouragement. Besides thanking Weil, the author also wishes to thank J.-L. Verdier for helpful conversations which took place at the Universidad Nacional Autónoma de México during the summer of 1971.

Let $k$ be a $p$-field with residue field $\boldsymbol{F}_{q}$ and ring of integers $r$. Let $V(\beta)$ be a nontrivial Severi-Brauer $k$-scheme of dimension $n-1$. Using local class field theory, a canonical $r$-scheme $V_{r}(\beta)$ can be constructed having generic fiber birationally isomorphic to $V(\beta)$. Explicitly, let $k_{n}$ denote the unramified extension of $k$ of degree $n, G=\operatorname{Gal}\left(k_{n} \mid k\right)$; let $\zeta$ be a primitive $q^{n}-1$ root of unity in $k_{n}$ and $\pi$ be a prime element of $k$. Let $M=$ $r\left[X_{0}, X_{1}, \cdots, X_{n}\right] / I(\beta)$, where $I(\beta)$ is the ideal generated by

$$
\prod_{g \in G}\left(X_{0}+g(\zeta) X_{1}+g\left(\zeta^{2}\right) X_{2}+\cdots+g\left(\zeta^{n-1}\right) X_{n-1}\right)-\pi^{s} X_{n}^{n}
$$

and $s$ is an integer (determined by $\beta), 1 \leqq s \leqq n-1$. Then $V_{r}(\beta)=\operatorname{Proj}\left(M^{\sim}\right)$. The closed fiber of $V_{r}(\beta)$ is a union of hyperplanes conjugate by elements of $\operatorname{Gal}\left(\boldsymbol{F}_{q^{n}} / \boldsymbol{F}_{q}\right)$. Its zeta function is

$$
\left[\left(1-\left(q^{n-1} T\right)^{n}\right)\left(1-\left(q^{n-2} T\right)^{n}\right) \cdots\left(1-(q T)^{n}\right)(1-T)\right]^{-1} .
$$

$V_{r}(\beta)$ will be called the canonical model for $V(\beta)$. In case $V(\beta)$ is a trivial Severi-Brauer $k$-scheme, two types of models for $V(\beta)$ over Spec $r$ can be constructed, each having "good" properties under specialization. In particular, the zeta function of the closed fiber of each of these models is

$$
\left[\left(1-q^{n-1} T\right)\left(1-q^{n-2} T\right) \cdots(1-T)\right]^{-1} .
$$


From now on let $k$ and $r$ denote, respectively, an algebraic number field and its ring of integers. Let $\Omega$ be the set of places of $k$. For $v \in \Omega$, let $k_{v}$ denote the completion on $k$ at $v$ and, in case $v$ is finite, $r_{v}$ the ring of integers in $k_{v}$. Let $V(\beta)$ be a Severi-Brauer $k$-scheme of dimension $p-1, p$ a prime. Let $\Omega_{0}$ denote the set of $v \in \Omega$ for which $V\left(\beta_{v}\right)=V(\beta) \times_{k}$ $k_{v}$ is a nontrivial Severi-Brauer $k_{v}$-scheme; $\Omega_{0}$ is finite. Using the GrunwaldWang theorem [4], strong approximation, and "recollement de faisceaux" a coherent sheaf $\mathscr{S}$ of $o_{r}$-algebras flat over $o_{r}$ can be constructed so that the $r$-scheme $X=\operatorname{Proj}(\mathscr{S})$ has the following properties:

(a) $X \times_{\text {spec } r} k$ is birationally isomorphic to $V(\beta)$.

(b) For all $v \in \Omega_{0}, X_{v}=X \times \times_{\text {spec } r} \operatorname{Spec} r_{v}$ is isomorphic as an $r_{v}$-scheme to the canonical model for $V(\beta)$.

(c) For all $v \in \Omega-\Omega_{0}, X_{v}$ is isomorphic as an $r_{v}$-scheme to one of the two models for trivial Severi-Brauer $k_{v}$-schemes.

Let $\gamma$ be the image of $\beta$ in $\operatorname{Br}(k)$ and $D(\gamma)$ the division algebra defined by $\gamma$. Let $Z_{D}$ denote the zeta function of $D(\gamma)$ [2], [11] and $Z_{X}$ denote the zeta function of $X$ in the sense of [9] with factors corresponding to the archimedean primes as defined in [8]. $Z_{D}$ and $Z_{X}$ have the same local factors corresponding to all $v \in \Omega-\Omega_{0}$. Hence the Euler product defining $Z_{X}$ converges absolutely for $s \in C, \operatorname{Re}(s)>p . Z_{X}$ can be continued analytically to a meromorphic function on the complex plane with simple poles at $s=0, p$. Let $N \delta$ denote the norm of the different of $D(\gamma)$ over $k$ and $D$ denote the discriminant of $k$ over $Q$; then an easy calculation using the results of [10] yields the functional equation

$$
Z_{X}(s)=(N \delta)^{(s / p-1 / 2)(p-1)}|D|^{p(p / 2-s)} Z_{X}(p-s)
$$

\section{REFERENCES}

1. A. A. Albert, Structure of algebras, Amer. Math. Soc. Colloq. Publ., vol. 24, Amer. Math. Soc., Providence, R.I., 1939. MR 1, 99.

2. M. Deuring, Algebren, Zweite, korregierte Auflage, Ergebnisse der Mathematik und ihrer Grenzgebiete, Band 41, Springer-Verlag, Berlin and New York, 1968. MR 37 \#4106.

3. S. A. Amitsur, Generic splitting fields of central simple algebras, Ann. of Math. (2) 62 (1955), 8-43. MR 17, 9.

4. E. Artin and J. Tate, Class field theory, Benjamin, New York, 1968. MR 36 \#6383.

5. F. Châtelet, Variations sur un thème de H. Poincaré, Ann. Sci. École Norm. Sup. (3) 61 (1944), 249-300. MR 7, 323.

6. P. Roquette, On the Galois cohomology of the projective linear group and its applications to the construction of generic splitting fields of algebras, Math. Ann. 150 (1963), 411-439.

7. J.-P. Serre, Corps locaux, Hermann, Paris, 1968.

8. - Facteurs locaux des fonctions zêta des variétés algébriques (définitions et conjectures), Séminaire Delange-Pisot-Poitou, 1969/70, exposé 19. 
9. J.-P. Serre, Zeta and L-functions, Arithmetical Algebraic Geometry, Harper and Row, New York, 1965, pp. 82-92. MR 33 \#2606.

10. S. Turner, Zeta-functions of central simple algebras over global fields, Dissertation, Brandeis University, Waltham, Mass., 1970.

11. A. Weil, Basic number theory, Die Grundlehren der math. Wissenschaften, Band 144, Springer-Verlag, Berlin and New York, 1967. MR 38 \#3244.

School of Mathematics, Institute For Advanced Study, Princeton, New Jersey 08540

Departamento de Matemática, Pontifícia Universidade Católica do Rio de JANEIRo, BrazIL (Current Address) 